\title{
LA AUTONOMÍA PERSONAL Y LA AUTONOMÍA RELACIONAL
}

\author{
Silvina ÁlvAREZ
}

Facultad de Derecho, Universidad Autónoma de Madrid

\section{Resumen}

Muchos autores que se han ocupado de la autonomía personal parecen identificar la capacidad de autonomía esencialmente con la capacidad de todo agente racional para identificar preferencias y tomar decisiones conforme a las mismas. Estos autores -entre los que podríamos ubicar a C. Nino- prescinden a menudo de ulteriores elementos o condiciones para su ejercicio. Sin embargo, resulta fundamental ahondar en esos aspectos a veces pasados por alto; ahondar principalmente en las opciones y el proceso de formación de preferencias de los agentes, para delinear mejor los aspectos normativos de la autonomía. En este trabajo propongo recuperar un elemento central del concepto que ha sido extensamente desarrollado en la literatura feminista, me refiero al contexto de relaciones que ha dado lugar a la llamada "autonomía relacional". Propongo incorporar esta noción al núcleo conceptual de la autonomía sin renunciar, sin embargo, a otros elementos del concepto entre los que destacaré las "opciones relevantes", es decir, la presencia de cursos de acción, de oportunidades, que la persona es capaz de reconocer como propuestas no solo viables sino legítimas para sí.

Para desarrollar esta idea comenzaré por definir la autonomía a través de los que considero los elementos o condiciones centrales del concepto, deteniéndome especialmente en la condición de opciones relevantes. A continuación se abordará la cuestión relativa al modo en que las opciones se perfilan, se construyen y son percibidas por los sujetos. En este punto el estudio se fijará específicamente en el entorno contextual y de relaciones que sirve de trasfondo para la construcción de las opciones, para proponer la noción de opciones relacionales.

PALABRAS CLAVE: Autonomía personal; Autonomía relacional; Racionalidad; Independencia; Oportunidades; Opciones; Contexto social; Contexto de relaciones.

\begin{abstract}
Many authors have written about autonomy focusing mainly on the capacity of the autonomous agent for identifying preferences and making decisions. These authors $-\mathrm{C}$. Nino among them- do not take into consideration further elements or conditions for being autonomous. However, it is necessary to take other aspects into consideration; mainly it is important to take account of both options and the building of the agent's preferences. This way, autonomy normative aspects will be better understood. I propose here to analyse the concept of "relational autonomy", which has been largely elaborated within feminist theory. My aim is to incorporate relations and options to the core of the concept of personal autonomy.

In this article, I will define personal_autonomy first, highlighting the importance of relevant options. Second, I will describe the way options are built and their perception
\end{abstract}


by the agent. Finally, I will focus on the context and relations on the background, and I will propose the notion of relational options.

KEY WORDS: Personal Autonomy; Relational Autonomy; Independence; Opportunities; Options; Social Context; Relational Context.

La autonomía personal es un concepto fundamental de la filosofía moral, de la filosofía política, del derecho y también de la teoría feminista. Las razones por las que se han dedicado y se siguen dedicando tantos esfuerzos a perfilar el concepto tienen que ver con el entramado de valores en el que la autonomía se enmarca y a cuya realización contribuye. Estos valores apuntan seguramente al ideal de la emancipación, del autogobierno; a la realización de la libertad individual a través del ejercicio consecuente de la racionalidad práctica; a la fundamentación de su contrapartida, la responsabilidad; a la igualdad entendida como consideración y respeto por las elecciones individuales; a la diferencia que emana de las decisiones de las personas. Todas estas aspiraciones giran de alguna manera en torno al concepto de autonomía y por esto resulta tan importante precisar el concepto. Pero junto a los ideales y valores a los que se asocia la autonomía, la tarea de construcción del concepto cobra importancia también, y tal vez sobre todo, por aquello que como concepto normativo quiere rechazar. La autonomía se opone a los modelos de dominación y opresión; condena la imposición heterónoma de principios y normas de conducta; rechaza la dependencia producto de negar reconocimiento moral a las personas, rechazando de este modo la discriminación y la marginación; condena el autoritarismo en la medida en que representa la negación de la capacidad de elección política. Este entramado valorativo en que se enmarca la autonomía no debe entenderse, sin embargo, como un avance de lo que podría ser una concepción sustantiva o perfeccionista, sino como el escenario en el cual nos movemos y sin el cual no se puede entender la importancia de la autonomía como valor o principio universal.

La centralidad del concepto de autonomía personal no escapó a la obra de Carlos Nino. Tanto en la construcción del concepto de derechos individuales como en su propuesta filosófico-moral, la autonomía personal constituye un trasfondo manifiesto de su pensamiento, en sintonía con el resto de presupuestos liberales que forjan su pensamiento. En su Ética y derechos humanos encontramos una caracterización de la concepción de autonomía personal que Nino utiliza. En constante contrapunto con los límites del poder estatal y con la posible deriva perfeccionista de dicho poder, Nino se decanta por una concepción formal de la autonomía: "[...] las acciones que están determinadas por la libre 
adopción de tales principios, tienen algún valor prima facie. Esto refleja lo que suele calificarse como el valor de la autenticidad moral y hace eco a la idea de que aun en las acciones de un nazi convencido puede haber algún valor." Aunque su concepción se centre claramente en la capacidad de elección o la capacidad para escoger planes de vida, no se detiene en un análisis minucioso de los elementos o condiciones necesarias para el ejercicio de la autonomía. Aunque cite a Raz, ${ }^{2}$ tampoco profundiza en las opciones como parte del concepto de autonomía. Y aunque se refiere a las preferencias del agente y a su papel en la concreción de la autonomía, no se fija en los procesos de formación de tales preferencias. Parecería que Nino identifica la capacidad de autonomía esencialmente con la capacidad de todo agente racional, prescindiendo de ulteriores elementos o condiciones para su ejercicio.

Creo, sin embargo, que es importante ahondar en esos aspectos a veces pasados por alto, es decir, las opciones y el proceso de formación de preferencias de los agentes, para delinear mejor los aspectos normativos de la autonomía, en sintonía con el ideario o entramado de valores que mencionaba más arriba. En esta tarea, la teoría feminista ha hecho en los últimos años importantes aportes al concepto de autonomía personal. No solo se ha encargado de sopesar el lugar que el concepto puede o debe tener en relación con las reivindicaciones de las mujeres, sino que ha contribuido al debate teórico sobre el concepto, su alcance y los elementos que lo definen. En este trabajo propongo recuperar un elemento central del concepto que ha sido extensamente desarrollado en la literatura feminista; me refiero al contexto de relaciones que ha dado lugar a la llamada "autonomía relacional". Propongo incorporar esta noción al núcleo conceptual de la autonomía sin renunciar, sin embargo, a otros elementos del concepto entre los que destacaré las "opciones relevantes", es decir, la presencia de cursos de acción, de oportunidades, que la persona es capaz de reconocer como propuestas no solo viables sino legítimas para sí.

Para desarrollar esta idea comenzaré por definir la autonomía a través de los elementos o condiciones que considero centrales del concepto, deteniéndome especialmente en la condición de opciones relevantes. A continuación se abordará la cuestión relativa al modo en que las opciones se perfilan, se construyen y son percibidas por los sujetos. En este punto el estudio se fijará específicamente en el entorno contextual y de relaciones que sirve de trasfondo para la construcción de las opciones, para proponer la noción de opciones relacionales.

${ }^{1}$ Nino (1989, p. 234).

${ }^{2}$ Véase Nino (1989, pp. 207, 208, 213, 226). 


\section{El concepto de autonomía}

Empezaré entonces por trazar una breve descripción de los que considero los rasgos fundamentales del concepto. La autonomía es una capacidad de las personas y, como tal, admite desarrollos variados que pueden condicionar fuertemente su ejercicio. Esto hace que sea tan difícil afirmar de alguien que no tiene, en absoluto, autonomía, como afirmar que tiene una autonomía máxima. Tener más o menos autonomía depende de una serie de factores, de condiciones internas y externas al sujeto. Entre las condiciones internas la literatura clásica sobre la autonomía ha destacado la racionalidad. ${ }^{3}$ Esta condición, netamente kantiana en su formulación originaria, ha sido enriquecida por ulteriores desarrollos relativos a las posibilidades de la acción racional, en la medida en que va precedida de un proceso de singularización de los deseos o preferencias, seguido de otro proceso de jerarquización de las mismas. ${ }^{4}$ Esta compleja tarea que cada sujeto realiza comprometiendo su capacidad de reflexión comporta evaluar, calibrar, sopesar y finalmente asignar un orden de prioridades; orden que, a su vez, responderá a pautas que revelan la disposición moral y emocional del sujeto. ${ }^{5}$

Este proceso que implica necesariamente la psicología del agente racional, se complementa con otra condición también central, que es la independencia. Mientras que la racionalidad hace referencia a una condición estrictamente subjetiva, exclusivamente interna del sujeto, la independencia se mueve en un terreno dual. Por un lado, también la independencia es una condición interna en la medida en que se refiere a la aptitud del sujeto para distanciarse de influjos ajenos, de condicionamientos externos, de deseos y preferencias que no son las suyas. Esto no quiere decir que las preferencias no puedan configurarse fuera del sujeto sino que para considerarlas propias las tiene que haber querido

${ }^{3}$ Esta condición ha sido expresada de muy diversas maneras en la bibliografía sobre autonomía. Algunos trabajos se refieren a la capacidad de reflexión, otros a la capacidad de análisis y otros más generalmente a las aptitudes mentales del agente. Véase Raz (1986), Dworkin (1988), Young (1986).

${ }^{4}$ Sobre autonomía personal y estratificación de preferencias véase Frankfurt (1971).

${ }^{5}$ Hablo aquí de disposición moral y emocional, entendiendo que el sujeto razona en sintonía con sus emociones, de las que no solo no puede desprenderse completamente (aunque sí pueda esforzarse por atemperarlas o quitarles protagonismo), sino que forman parte necesaria de la agencia moral. La relación entre autonomía y emociones requiere de un estudio aún pendiente en la literatura sobre la materia. Sobre las emociones y sus aspectos cognitivos y racionales, véase Oakley (1992, pp. 6-121); véase también Griffiths (1995, pp. 97-108). 
y escogido para sí. La independencia es en este sentido la aptitud para decidir por una misma, para no dejar en manos de otras personas elecciones relevantes. Por otro lado, la independencia tiene un aspecto externo en la medida en que nos habla de la posición que el sujeto ocupa respecto de su entorno y del tipo de relación que tiene con las personas con las que interacciona. Es decir, este segundo sentido de independencia toma en cuenta el contexto y las relaciones, el trasfondo de significado del que se nutre la capacidad emocional, cognitiva y conductual de la persona. En este sentido, se hace necesario recordar que numerosos aspectos de la vida de la persona vienen condicionados por circunstancias que no están sujetas a decisión ni a revisión personal. Circunstancias, como las características físicas del sujeto (sexo, color de piel, etnia, etc.), que no solo quedan fuera del ámbito de revisión sino que conforman un marco de referencia que puede condicionar de manera importante el contenido de las decisiones personales y del que difícilmente pueda el sujeto autónomo independizarse. Como apunta John Christman, dichas circunstancias van acompañadas de "significados sociales" sobre los que en algunos casos no resulta fácil intervenir. Todo esto debe ser tomado en cuenta para evaluar la verdadera extensión de la independencia y del campo de decisión del agente. ${ }^{6}$

En otras palabras, la condición de independencia debe plantearse incluyendo, en primer lugar, la capacidad de la persona para reflexionar sobre sí misma, el entorno y las otras personas. En segundo lugar, dicha capacidad debe unirse a la aptitud para tomar distancia respecto del entorno de relaciones y significado dado. Esta doble dimensión de la independencia es necesaria para que el sujeto cobre conciencia de su posición, de la distancia que lo vincula al entorno y a los otros. Por último, la condición de independencia debe entenderse como la capacidad para tomar decisiones sobre la base de las propias preferencias, habida cuenta del reconocimiento directo o indirecto del entorno y las relaciones (volveremos sobre esta cuestión). Así definida la condición de independencia, y en contra de lo que sugiere la concepción clásica de la autonomía como independencia, ${ }^{7}$ vemos entonces

${ }^{6}$ Véase Christman (2001, pp. 185-206). Siempre en relación con los aspectos descriptivos o fácticos de la autonomía, el desarrollo y formación de las preferencias y la relevancia de una perspectiva histórica de la autonomía, véase Christman (1991, pp. 1-24), Mele (1993, pp. 271-280), Christman (1993 pp. 281-289); sobre el enfoque empirista y las preferencias reveladas, véase también O’Neill (1992, pp. 205-213).

7 Sobre este particular véase la crítica de Nedelsky a la autonomía como independencia (Nedelsky 2011, p. 118). 
que encierra una importante dimensión relacional. ${ }^{8}$ Ser independiente no es apartarse o aislarse de los demás, sino más bien lo contrario: tomar en consideración el entramado de relaciones en el que nos desarrollamos para poder posicionarnos, definirnos y decidir cuál es nuestra propia disposición en ese marco.

Se hace necesario ahora introducir otra condición importante en la configuración de la autonomía. Joseph Raz (1986) se ha referido a las opciones relevantes como condición necesaria para la autonomía: quienes no tienen ante sí una gama de opciones suficientemente importantes (no triviales ni delimitadas por la urgencia de la supervivencia) no están en condiciones de ejercer la autonomía. ${ }^{9}$ No pueden ejercerla porque esta consiste precisamente en la capacidad para tomar decisiones que afectan significativamente la vida del sujeto y que por tanto deben reflejar las preferencias de la persona. Si las opciones son insuficientes o inadecuadas no se dan las condiciones para que las preferencias puedan expresarse. Tal como las presenta Raz, las opciones se refieren a un componente de la autonomía que es externo al sujeto y que permitiría entonces hacer una evaluación de la misma a partir de elementos objetivos que tienen que ver precisamente con el contexto y las relaciones en las que se inscribe la acción del sujeto. ${ }^{10}$ Nino se refiere indirectamente a estas cuestiones que plantea Raz, cuando menciona la necesidad de "bienes públicos o colectivos" que en la concepción raziana permitirían un ejercicio efectivo de la autonomía (1989, p. 226). Asimismo, Nino esboza algunas objeciones a este concepto, sobre todo en relación con los problemas de escasez, distribución y conflictos que puede plantear la adjudicación de dichos bienes (1989, p. 228).

La introducción de las opciones en el concepto de autonomía tiene un significado muy importante, en la medida en que comporta el reconocimiento de que no se trata de una capacidad cuya presencia o no en el agente pueda valorarse solo a partir de constatar que concurren las condiciones que antes identificamos como racionalidad e independencia, en su sentido subjetivo o interno. Al introducir las opciones como elemento de la autonomía, se señala que se trata de una capacidad cuyo ejercicio

${ }^{8}$ Haworth menciona dos aspectos relacionales de la independencia vinculados al conocimiento que el sujeto tiene de sí mismo, por un lado, y de los demás, por otro; asimismo, resalta la relevancia de la independencia en relación con la acción (Haworth 1986, p. 13).

${ }^{9}$ Conviene observar que, así configurada la condición de relevancia de las opciones, la autonomía se define, como he sostenido en otra parte, no por los resultados sustantivos de su ejercicio sino por las condiciones sustantivas para su ejercicio (Álvarez 2002, pp. 158-159).

${ }^{10} \mathrm{Raz}$ (1986, pp. 373-390). 
está en función del tipo de estímulos, incentivos, situaciones, relaciones o entorno con que se enfrenta el agente. Las opciones se configuran precisamente a partir de todos aquellos factores que conforman el escenario de toma de decisiones de una persona. El ámbito familiar y de relaciones afectivas que rodean el desarrollo evolutivo en la infancia marcan sin duda la autonomía de los niños y niñas, en la medida en que podemos hablar de una incipiente autonomía. ${ }^{11}$ Progresivamente, el entramado de relaciones traspasa el ámbito familiar para abarcar contextos más extensos y variados. Así, las opciones de una persona adulta darán cuenta de la red de relaciones de la que forma parte, así como de los ámbitos o contextos -familiar, de amistades, social, laboral, etc.- en los que actúa e interactúa. Todo este entramado relacional y contextual que rodea al agente tiene una dimensión objetiva, externa, observable. Pero dicho entramado dota a las opciones también de un significado subjetivo que depende del tipo de inserción que vincula al agente con otras personas en su ámbito de referencia.

Así, existen opciones en la medida en que se dan tanto las condiciones externas (en relación con el contexto y relaciones), es decir las oportunidades, como las condiciones internas (en relación con la percepción que el sujeto tiene del contexto y la propia inserción en el entramado de relaciones). Dicho con otras palabras, las opciones para configurarse como tales requieren que las oportunidades sean percibidas por el agente como legítimas y viables para sí; solo de este modo un curso de acción podrá ser identificado, seleccionado y llevado a cabo por el agente autónomo. ${ }^{12}$ Volveré más adelante sobre este particular.

Se podría objetar, sin embargo, que queda por delimitar el umbral a partir del cual se puede considerar que las opciones que tiene ante sí el agente son lo suficientemente relevantes, no distorsionadas, por ejemplo, por relaciones de subordinación o antecedentes de discriminación, como para potenciar la autonomía de los sujetos. Me parece importante resaltar aquí las dificultades para clasificar, sin más, los contextos adversos como escenarios en los que las personas no son autónomas. Se ha señalado ya que la capacidad de autonomía es una competencia gradual y que por tanto se puede predicar de las personas

11 Sobre el desarrollo de la autonomía en los primeros estadios de la infancia y lo que el autor denomina "autonomía mínima”, véase Haworth (1986,pp. 16-21).

$12 \mathrm{El}$ aspecto subjetivo de las opciones tal como aquí las he presentado, parece tener puntos de contacto con lo que en el análisis de J. Nedelsky se define como el "sentimiento de autonomía" o la posibilidad de autopercibirse como un agente capaz de tener la experiencia de actuar autónomamente (Nedelsky 2011, p. 135). 
en mayor o menor medida en función de cómo se presenten los elementos o condiciones para su ejercicio. Pero esto nos enfrenta con la necesidad de establecer, en primer lugar, en qué medida o extensión mínima se deben verificar las tres condiciones que aquí se han señalado -racionalidad, independencia y opciones relevantes- para que pueda existir autonomía; y en segundo lugar, en qué medida dichas condiciones no comprometen contenidos sustantivos y, por tanto, en qué medida nos encontramos ante una propuesta perfeccionista. ${ }^{13}$ Lamentablemente no podré extenderme aquí sobre estas cuestiones ${ }^{14}$; profundizaré algo más, en cambio, sobre el concepto de autonomía, las opciones y su contexto relacional.

\section{Las opciones relacionales}

Volvamos a las opciones. En la caracterización que se ha realizado de la autonomía personal, el elemento que marca seguramente un giro importante respecto de la concepción clásicaliberal de la autonomía es el relativo a las opciones, tanto en la vertiente objetiva como subjetiva del concepto. En el primer caso porque implica admitir que la autonomía no se dirime solo en primera persona, que no es solo un indicador de las habilidades cognitivas del sujeto racional, sino que el desarrollo de dichas capacidades está fuertemente condicionado por elementos externos al sujeto, por el contexto y por las relaciones que en el marco de dicho contexto el sujeto entabla. En el segundo caso porque se señala la fuerte interrelación que existe entre los aspectos cognitivos y los psicológicos, y entre estos y el contexto de oportunidades y relaciones. En ambas vertientes (objetiva y subjetiva) de las opciones, la forma en que el sujeto se vincula con el entorno y el tipo de relaciones que entabla con otros sujetos resultan centrales para determinar el contenido y extensión de lo que aquí llamaré opciones relacionales.

13 Como he sostenido en otro lugar, un concepto de autonomía que incluya las opciones como condición de ejercicio comporta necesariamente una valoración del contenido de las opciones y, por tanto, del contexto de ejercicio; no comporta, sin embargo, una valoración del contenido de las elecciones efectivas de los sujetos. En otras palabras, introducir la condición de las opciones adecuadas nos desplaza hacia el terreno de una autonomía definida no por los resultados sustantivos de su ejercicio pero sí por las condiciones sustantivas para su ejercicio (Álvarez 2002, pp. 158-160).

${ }^{14}$ Para un tratamiento ulterior de las mismas, véase Álvarez (2014). 


\section{El contexto de relaciones}

Annette Baier, en un artículo muy citado por quienes en los últimos años han propuesto un concepto relacional de autonomía ${ }^{15}$, afirma que "las personas son esencialmente segundas personas"16. Esta afirmación apunta en primer lugar a la psicología evolutiva, a la necesidad de interacción que tienen las niñas y niños para desarrollar sus capacidades cognitivas, emocionales y conductuales. "Las personas vienen después y antes de otras personas"; "Las personas son esencialmente sucesoras, herederas de otras personas que las formaron y cuidaron de ellas" (1985a, p. 85). Este origen que señala la dependencia de los humanos de otros humanos, sirve a la autora para señalar una interpretación del sujeto cartesiano que va más allá de la interpretación al uso de los sujetos cartesianos como exclusiva conciencia racional. La conciencia cartesiana en un sentido más amplio se traslada de la "autoconciencia" a una conciencia que se reconoce en relación con otras personas:

Las personas son autoconscientes, saben que son personas entre las personas. [...] Mi primer concepto de mí misma es como el referente de "tú", pronunciado por alguien a quien yo me voy a dirigir como "tú" (Baier 1985a, p. 89).

Estas afirmaciones no deben interpretarse en el sentido de anclar al individuo en su entorno, en el determinismo cultural o social o, incluso, en el esencialismo. Cualquiera sea la forma y el contenido que estas relaciones puedan adquirir en cada caso, ser relacionales es un aspecto de la psicología de los individuos del que no podemos prescindir y que por tanto debemos tener en cuenta en la elaboración de los conceptos con los que queremos analizar los comportamientos de las personas. Mi intención es, por tanto, rescatar de esta concepción un punto de partida, un escenario no solo descriptivo sino también normativo, con el que debemos contar: las personas se constituyen como tales en un contexto de interdependencia. ${ }^{17}$

15 Véase, entre otras, Code (1991, pp. 74, 82, 84-87, 90, 115, 116, 122, 126, 270, 280), Mackenzie y Stoljar (2000, p. 7), Brison (2000, p. 283).

${ }^{16}$ Baier (1985a, p. 77).

17 Rescatar este punto de partida significa también redefinir, reposicionar la autonomía o, dicho de otro modo, agregar un elemento o condición para su ejercicio. No entiendo aquí que esta nueva condición reste importancia a la autonomía sino que la ubica en el contexto de socialización en el que las personas desarrollan y ejercen su capacidad de toma de decisiones. Para un análisis de la autonomía de las mujeres a partir de la propuesta de Baier, véase Code (1991, pp. 82-87). 
Esta afirmación resulta muy importante para el análisis de la autonomía personal ya que, incluso si aceptamos que la autonomía personal reivindica precisamente la capacidad de las personas para revertir los resultados de dicha interdependencia o incluso la interdependencia misma, no podemos dejar de reconocer un escenario relacional que sirve de trasfondo necesario para las decisiones autónomas.

Con las herramientas forjadas durante su desarrollo evolutivo, las personas adultas siguen actuando en un contexto fuertemente relacional, en el que tanto las posibilidades de acción como la percepción y elección finales se dirimen relacionalmente. Esto no debe confundirse, insisto, con el esencialismo (social o cultural). No se está diciendo que dicho entramado relacional merme o anule las posibilidades de toma de decisiones de los sujetos. Lo que pone en evidencia dicho entramado intersubjetivo es la peculiar imbricación que nuestras decisiones tienen. Una imbricación que revela de qué manera se ponen en práctica nuestras capacidades como sujetos racionales. En primer lugar, el sujeto racional construye su independencia que, como hemos dicho ya, tiene un fuerte elemento relacional. Construir la independencia significa tomar distancia y reubicar, según nuestra propia perspectiva y evaluación, los influjos externos para poder entonces ordenar las propias preferencias. En segundo lugar, el sujeto racional debe sopesar las opciones que tiene a su alcance para poder actuar. Todo esto tiene lugar en un marco relacional que confiere al sujeto una posición relativa, es decir, un lugar en relación con las otras personas de su entorno. ${ }^{18}$

Dicho marco relacional puede entenderse como una dimensión de lo que algunas autoras han estudiado en términos de "socialización". Un

\footnotetext{
${ }^{18}$ En este sentido resulta pertinente lo que apunta Oshana sobre la necesidad de que la persona autónoma sea consciente del entramado social del que forma parte (Oshana 2005, pp. 92-93). Oshana afirma que la persona autónoma debe ser consciente de la influencia que ejercen sobre ella -tanto que pueden condicionar o disminuir su propia autonomía- las relaciones sociales que ayudan a perfilar su identidad (2005, p. 94).

Esta condición de Oshana está abocada a dejar fuera de la esfera de autonomía numerosas decisiones individuales de quienes se han visto sometidas a procesos de socialización más restrictivos de la libertad. Desde una perspectiva procedimental de la autonomía, tenderíamos a considerar las decisiones de quienes no tienen ese grado de autoconciencia sobre su propia identidad como autonomías no plenas o menos desarrolladas, sin despreciar, sin embargo, la capacidad de toma de decisiones. Un aspecto interesante en relación con la autoconciencia es el relativo a si la autonomía necesitaría también de cuotas importantes de autoconfianza o confianza en una misma para poder concebir decisiones independientes. Sobre autoconfianza y autonomía véase Govier (1993, pp. 111-112). Para un análisis de la posición de Oshana en relación con el umbral de autonomía, véase Álvarez (2014).
} 
buen ejemplo de esta perspectiva es el estudio de Meyers, quien antes de ocuparse de las posibilidades de la autonomía se interroga sobre la influencia que los factores sociales ejercen sobre la psicología de los individuos -o, lo que es lo mismo, sobre el influjo más o menos determinante de la sociedad sobre el individuo-(Meyers 1989, pp. 25-41). Resaltando la relevancia de la sociedad en la conformación de numerosos aspectos de la psicología de las personas, la autora reivindica la necesidad de analizar con detenimiento el contexto social de referencia para comprender mejor las posibilidades de la autonomía como competencia (1989, pp. 135-136). Asimismo, Diana Meyers se ocupa de cómo la distinta socialización de varones y mujeres promueve mayores cuotas de autonomía en los primeros que en las segundas. No escapan del análisis de la autora los estudios de Nancy Chodorow sobre la psicología femenina ni las consideraciones relativas a la importancia de la educación para la autonomía (1989, pp. 136; 172; 192-202).

Al hablar de relaciones, entonces, no nos referimos solamente, aunque también, a las concretas relaciones que cada uno entabla a lo largo de su vida, relaciones afectivas, familiares, conyugales, fraternales, amistosas, laborales, etc. Sin duda estas relaciones afectan fuertemente nuestra vida y van conformando de manera directa o indirecta el espectro de opciones que tenemos ante nosotros al momento de tomar decisiones relevantes. Junto con estas concretas relaciones, los sujetos participan de formas menos específicas, más difusas, tal vez más impersonales en el sentido que se trata de relaciones no-nominales, con sujetos que no identificamos por sus nombres sino por la posición que ocupan en el contexto en el que nos desarrollamos y actuamos. Todo esto, sin embargo, no hace que este último tipo de relaciones, podríamos decir estandarizadas, sea menos relevante en la conformación de las opciones relacionales que abonan la noción autonomía. Por el contrario, estos entramados de relaciones predefinidas, con las que contamos y presuponemos para llevar a cabo nuestras acciones, ejercen un condicionamiento importante. Se trata de relaciones estereotipadas que nos indican en qué direcciones podemos avanzar. Es el tipo de radiografía previa que nos hace acercarnos a distintos ámbitos con distintas disposiciones psicológicas o motivacionales. Y estas relaciones así inscritas en sus contextos van acompañadas, a su vez, de un conjunto de motivaciones, disposiciones, actitudes de quienes participan en ellas.

En síntesis, nos hemos centrado en la reformulación de las condiciones para la autonomía: racionalidad, independencia relacionalcontextual y opciones relacionales relevantes. Si es cierto lo que aquí se 
ha sostenido sobre el papel de las oportunidades y las opciones para el ejercicio de la autonomía, los cambios que se produzcan en los aspectos contextuales y relacionales jugarán un papel central en la reconfiguración de las opciones. A su vez, se ha afirmado que la autonomía se desarrolla a través de la interacción que los aspectos más marcadamente internos o subjetivos -principalmente la racionalidad pero también la independencia- mantienen con los aspectos de mayor ascendencia externa u objetiva -principalmente las opciones-. Así, el concepto relacional de autonomía es capaz de tomar en cuenta los diversos aspectos que configuran el escenario de toma de decisiones. La mayor precisión conceptual que tal recuento arroja, nos permite calibrar mejor las consecuencias normativas tanto de la presencia de autonomía personal como de su ausencia. ${ }^{19}$

\section{Bibliografía}

Álvarez, S. (2002), "La autonomía personal”, en Díaz, E. y Colomer, J. L. (eds.), Estado, justicia, derechos, Madrid, Alianza, pp. 153-175. (2009), "Access to Opportunities in Multicultural Societies and the Relevance of Public Expression”, Social \& Legal Studies, 18(4), pp. 543-559.

(2014), "El umbral de autonomía", en Hierro, L. (ed.), Autonomía individual versus autonomía colectiva, Madrid, Marcial Pons, pp. 53-79.

Baier, A. (1985a), Postures of the Mind: Essays on Mind and Morals, Minneapolis, University of Minnesota Press.

_ 1985b), "What Do Women Want in a Moral Theory?", Nous, 19, pp. 53-64.

Brison, S. J. (2000), "Relational Autonomy and Freedom of Expression", en Mackenzie, C. y Stoljar, N. (eds.), pp. 280-299.

Chodorow, N. (1978), The Reproduction of Mothering: Psychoanalysis and the Sociology of Gender, Berkeley, University of California Press.

Christman, J. (1991), "Autonomy and Personal History," Canadian Journal of Philosophy, 21(1), pp. 1-24.

- (1993), "Defending Historical Autonomy: A Reply to Professor Mele," Canadian Journal of Philosophy, 23(2), pp. 281-289.

1995), "Feminism and Autonomy," en Bushnell, D. E. (ed.), "Nagging" Questions: Feminist Ethics in Everyday Life, Rowman \& Littlefield Publishers, pp. 17-39.

19 Sobre esta cuestión, véase Álvarez (2014, pp. 72-78). 
Christman, J. (2001), "Liberalism, Autonomy, and Self-Transformation", Social Theory and Practice, 27(2), pp. 185-206.

_ (2004), "Relational Autonomy, Liberal Individualism, and the Social Constitution of Selves", Philosophical Studies 117, pp. 143-164.

Christman, J. y Anderson. J. (eds.) (2005), Autonomy and the Challenges to Liberalism: New Essays, Cambridge University Press.

Code, L. (1991), What Can She Know? Feminist Theory and the Construction of Knowledge, Ithaca, Cornell University Press.

Dworkin, G. (1988), The Theory and Practice of Autonomy, Cambridge University Press.

Frankfurt, H. G. (1971), "Freedom of the Will and the Concept of a Person", The Journal of Philosophy, LXVIII(I), pp. 5-20.

Gilligan, C. (1982), In a Different Voice: Psychological Theory and Women's Development, Cambridge, Harvard University Press.

Govier, T. (1993), "Self-Trust, Autonomy, and Self-Esteem”, Hypatia, 8(1), pp. 99-120.

Griffiths, M. (1995), Feminism and the Self, Londres, Routledge.

Haworth, L. (1986), Autonomy: An Essay in Philosophical Psychology and Ethics, New Haven, Yale University Press.

Mackenzie, C. y Stoljar, N. (eds.) (2000), Relational Autonomy: Feminist Perspectives on Autonomy, Agency and the Social Self, Oxford University Press.

Mele, A. (1993), "History and Personal Autonomy," Canadian Journal of Philosophy, 23(2), pp. 271-280.

Meyers, D. T. (1989), Self, Society, and Personal Choice, New York, Columbia University Press.

Nedelsky, J. (2011), Law's Relations: A Relational Theory of Self, Autonomy, and Law, Oxford University Press.

Nino, C. S. (1989), Ética y derechos humanos, Buenos Aires, Astrea.

Oakley, J. (1992), Morality and the Emotions, Londres, Routledge.

O'Neill, O. (1992), "Autonomy, Coherence and Independence", en Miligan, D. y Miller, W. W. (eds.), Liberalism, Citizenship and Autonomy, Aldershot, Avebury, pp. 203-225.

(2005), "Autonomy and Self-Identity", en Christman, J. y Anderson, J. (eds.), pp. 77-97.

(2006), Personal Autonomy in Society, Aldershot, Ashgate.

Raz, J. (1986), The Morality of Freedom, Oxford University Press.

Stoljar, N. (2000), "Autonomy and the Feminist Intuition", en Mackenzie, C. y Stoljar, N. (eds.), pp. 94-111.

Taylor, J. S. (ed.) (2005), Personal Autonomy: New Essays on Personal Autonomy and Its Role in Contemporary Moral Philosophy, 
Cambridge University Press.

Young, R. (1986), Personal Autonomy: Beyond Negative and Positive Liberty, Londres, Croom Helm.

Recibido el 1 de septiembre de 2014; aceptado el 5 de diciembre de 2014. 